\title{
An Improved Positioning Algorithm Based on PSO and PLS
}

\author{
Hongmei Zhao ${ }^{1}$, Yanan $\mathrm{WU}^{2, \text { a }}$ \\ ${ }^{1}$ College of Electric and Information Engineering, Zhengzhou University of Light Industry, \\ Zhengzhou, 450000, China \\ ${ }^{2}$ College of Electric and Information Engineering, Zhengzhou University of Light Industry, \\ Zhengzhou, 450000, China \\ aemail:wyn629zq@163.com
}

Keywords: Ultra wide band;Indoor positioning; partial least squares;particle swarm optimization

\begin{abstract}
In order to realize the high precision positioning indoor based on the technology of ultra wideband,this paper proposes one kind of algorithm based on the partial least squares (PLS) and particle swarm optimization (PSO) algorithm, for the problem about the search ability of PSO algorithm low efficiency. Firstly, The PLS algorithm is used to evalute the initial estimation of position, knowing TOA and the real distance model. Then adhibiting PSO algorithm to optimize the initial position. The simulation results show that, the mean square error of the scheme is up to centimeter level positioning, while the Gauss error is 4. Analysis shows that this algorithm has strong adaptability to complicated deployment environment,and owns the advantage of stable performance, strong robustness.
\end{abstract}

\section{Introduction}

With the development of science and technology, "Location Services" has become the important basis of LBS and IOT for the fine development. Indoor commercial value is proportional to the positioning accuracy, UWB has a strong penetrating power, high time resolution, and the pulse width can reach ns or PS, can achieve centimeter level positioning.So it has become a hot research field.

This paper presents an improved algorithm to improve the positioning precision of the system. In 1983, S.Wold et al proposed a partial least squares (PLS) algorithm,in order to get mathematical modeling of the implicit function between the independent and dependent variables, decomposition and screening approach based on data mining system.The standard PSO algorithm is easy to understand, low complexity, maintenance is simple. But the late optimization problems are slow convergence speeding, easy to fall into local minima and premature convergence.

In this paper, for the first time, the PLS algorithm is applied to indoor positioning, put forward a kind of high precision UWB indoor location algorithm based on partial least squares regression. The simulation results show that: in the indoor environment, the algorithm can effectively overcome the shortcomings of PSO algorithm, with a high positioning accuracy.

\section{The Positioning Scheme Based on PLS and PSO}

The method is divided into two stages: the stage of training and positioning stage. In the training stage, train to the mapping of the real distance measurement, according to the known users and base station distance and physical distance learning, then establish a localization model; In the positioning stage, using mapping model training and the time of arrival (Time Of, Arrival, TOA) of the target to the base station, can estimate the position as shown in figure 1.

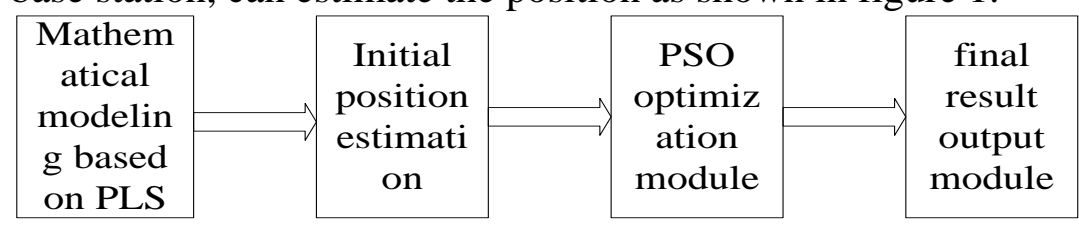

figure 1 . The overall flow chart of positioning system 
If the monitoring area into a two-dimensional plane, with the presence of $n$ base stations (including fixed base station and mobile station), the $m(m<n)$ is a known coordinate information base, the fixed base station BS. BS measurement data collected by TOA and the distance between BSs can be expressed by $T_{i}=\left[T_{i 1}, T_{i 2}, \cdots, T_{i m}\right]^{T}$ and $R_{i}=\left[R_{i 1}, R_{i 2}, \cdots R_{i m}\right]^{T}$. At this time, time of arrival (TOA) and the relationship between the real distance is expressed as:

$$
R=T \eta+\varepsilon
$$

Among them, $\eta$ is the regression coefficient , $\varepsilon$ is for the random error. In order to obtain the optimal linear relationship between TOA and distance. It is necessary to make $\|\varepsilon\|^{2}$ minimum, when the hour, can be

$$
T^{T} T \hat{\eta}=T^{T} R
$$

From the formula (1) set, $\boldsymbol{T}$ variables may be severe multiple correlation. While the equation (4) is force calculated, the estimation results will fail. In addition, the precision of estimated value not only with the input variables, but also output variables R.The details are as follows:

The initialization of $\mathbf{u}: \mathbf{u}=\mathbf{R I}$, where $\mathbf{R I}$ is an arbitrary $\mathbf{R}$ in a column vector; The weight vector of $w: w=\mathbf{T}^{\mathrm{T}} \mathrm{u} / \mathrm{u}^{\mathrm{T}} \mathrm{u}$ calculation $\mathbf{T}$; The normalized $\mathrm{t}: \mathrm{t}=\mathbf{X} \mathbf{w}, \mathbf{T}-\mathrm{t} / \mathrm{t}^{\text {"; }}$; The weight vector of $\mathrm{c}: \mathrm{c}=\mathbf{R}^{\mathrm{T}} \mathrm{t} / \mathrm{t}^{\mathrm{T}} \mathrm{t}$ calculation $\mathbf{R}$; The normalized $\mathrm{u}: \mathrm{u}=\mathbf{R} \mathrm{c}, \mathrm{u}-\mathrm{u} /$ "U"; If step 3 of $\mathbf{T}_{\mathrm{i}}{ }^{\mathrm{k}}$ is equivalent to $\mathrm{t}_{\mathrm{i}}{ }^{\mathrm{k}-1}$ (or the difference between the two in a predetermined range), the $\mathbf{T}_{\mathrm{i}}{ }^{\mathrm{k}}$ has been the convergence of its true value, it can perform step 7; otherwise, to step 2,the $(\mathrm{k}+1)$ th iteration;

When MS is away from the BS, $\mathrm{R}_{\mathrm{i}}{ }^{0}$ andR ${ }^{0}$ (the true distance) are close, use $\boldsymbol{B} \approx \boldsymbol{r}^{\mathrm{o}} \boldsymbol{I}$ about least square algorithm(LS), first for the estimation of $\mathrm{Za}$ :

$$
Z_{a}=\left[X, y, r_{1}\right]^{T} \approx\left(G_{a}^{T} Q^{-1} G_{a}\right)^{-1} G_{a}^{T} Q^{-1} h
$$

In the formula:

$$
G_{a}=\left[\begin{array}{lll}
\Delta x_{2} & \Delta y_{2} & \Delta r_{2} \\
\Delta x_{3} & \Delta y_{3} & \Delta r_{3} \\
\vdots & \vdots & \vdots \\
\Delta x_{m} & \Delta y_{m} & \Delta r_{m}
\end{array}\right] ; h=\frac{1}{2}\left[\begin{array}{c}
\Delta r_{2}^{2}-K_{2} \\
\Delta r_{3}^{2}-K_{3} \\
\vdots \\
\Delta r_{m}^{2}-K_{m}
\end{array}\right] ; Z_{a}^{0}=\left[\begin{array}{c}
x \\
y \\
r_{1}^{0}
\end{array}\right]
$$

$\mathrm{Q}=\mathrm{E}\left(\mathrm{nn}^{\mathrm{T}}\right)$ is noise vector, the approximate normal distribution. When the MS is near to the BS, use the formula (3) estimates $\mathrm{Za}$ again.

$$
\begin{aligned}
& Z_{a}=\left(G_{a}^{T} \Psi G_{a}\right)^{-1} G_{a}^{T} \Psi^{-1} h \\
& \Psi=B * Q * B, B=\operatorname{diag}\left(r_{2}^{\prime} \ldots r_{m}^{\prime}\right), r_{i}^{\prime}=Z_{a}(3)+\Delta r_{i}
\end{aligned}
$$

Particle swarm optimization algorithm is a new evolutionary algorithm, similarly to genetic algorithm,both are the kind of optimization algorithm based on iterative group. But it is more simple than the genetic algorithm, it has no crossover and mutation operation, the search for the optimal values of the current search to find the global optimal solution.

The main idea of this paper is: the initial position of PLS to obtain coordinates as the original position of the particle, the area around the BS can be used as the target area of it, through the iterative search for the optimal solution, until satisfactory results. The specific steps are as follows:

A: The partial least square regression equation is established, the not standardized regression coefficients were used as target variables to optimize;

B: Enter a new set of TOA values, use the model to gain the initial position of the user;

$\mathrm{C}$ : The initial position is esed as the particle, and the speed of the initial is seted. The fitness function is fixed. The definition of fitness function are as follows:

The definition of fitness function are as follows:

$$
\begin{aligned}
& f_{1}(x)=\frac{1}{m} \sum_{i=1}^{m}\left[\frac{\left|y_{m i}-y_{i}\right|}{y_{i}}\right] \\
& f_{2}(x)=\frac{1}{n} \sum_{i=1}^{n}\left[\frac{\left|y_{n i}-y_{i}\right|}{y_{i}}\right]
\end{aligned}
$$


Among them, $f_{1}(x)$ is the fitting error of sample data, $f_{2}(x)$ is reserved for the prediction error model number; $m$ is the number of samples, $n$ is used to predict the number of samples. $Y_{\mathrm{mi}}$ is the first $\mathrm{m}$ observations of fitted values, $\mathrm{y}_{\mathrm{Ni}}$ is the first $\mathrm{n}$ observations were used to predict the value of prediction, $\mathrm{Y}_{\mathrm{i}}$ is the value of observation in $I_{\text {th }}$ time. In order to find the optimal solution, (9) mested be reached minimum, which is the ultimate fitness function:

$$
\min f(x)=\alpha_{1} f_{1}(x)+\alpha_{2} f_{2}(x)
$$

Among them, $a_{1}$ and $a_{2}$ are the weight, $a_{1}+a_{2}=1$;

D. for each particle, compared the adaptation values of the best location of gbest and the value of experience ,if latter is better, it will be the best current position;

E. for each particle, the best fitness value position of the Pbest experience and global adaptation values are compared, if the good, the current global best position;

F.The speed and position of the particle is updated;

G. if the number of iterations to achieve the preset, executing step h; otherwise executing step d;

H. output for optimal results.

\section{The Simulation Results and Analysis}

In the study of the positioning algorithm bansed on location mode base station, software simulation method will be used to evaluate the performance of location algorithm commonly. In this section, through the simulation experiment to analyze and evaluate the performance of the UWB indoor localization based on PLS and PSO. Simulation on Matlab platform, all of the code is written using Matlab language. Taking account of the $10 \mathrm{~m} \times 10 \mathrm{~m}$ warehouse simulation diagram as test's experiment, four BSs are located in the four corners of the room. The parameters of particle swarm algorithm are as follows: the rate of particle is updated to Vmax=2.5, learning factor $\mathrm{C} 1$ and $\mathrm{C} 2$ are 1.5, the maximum weighted factor is 0.7368 , the minimum weighted factor is 0 , the population size of particle swarm into 20. In order to the stablility and accuracy of experimental results, each deployment simulation experiments are carried out 1000 times, the average error of the mean square root of the results as an evaluation basis. The experimental results shown in Figure 2 and figure 3.

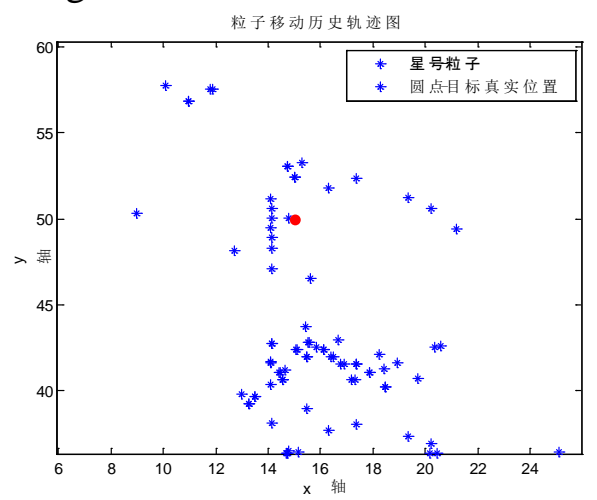

Fig.2. The positioning trajectory

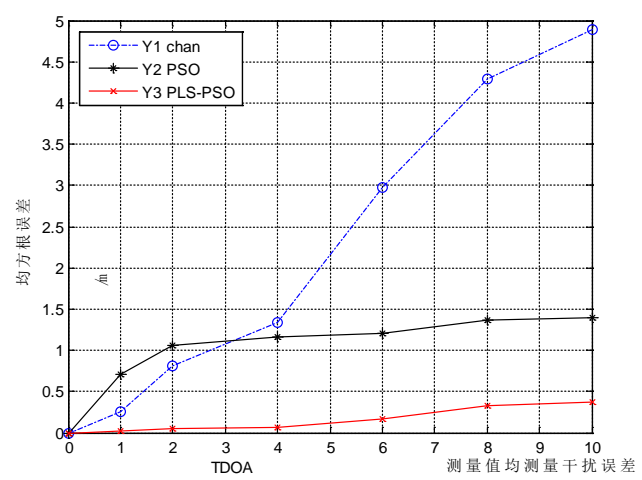

Fig.3. The experimental results

In Figure 3, depicts the process of particle iterative search to achieve high-precision positioning. The asterisk indicates the position of the particle experiences, red dots indicate the user real coordinates. From here we can see that the estimated coordinates of the algorithm, the final has been very close to the true value.

In figure 4. When the interference error of the Chan algorithm is small, the precision of positioning is high. But with the interference becomes larger, the positioning error is also growing. The PSO algorithm has little effect on the localization results of interference error. However, in order to achieve high precision location, PSO algorithm can not meet the requirements. UWB high precision positioning scheme is proposed in this paper, successfully combined to the PSO algorithm and Chan algorithm, overcome the PSO algorithm is slow convergence speed, easy to fall into local minima and so on shortcomings. 


\section{Conclusion}

This paper presents a high precision positioning scheme based on UWB technology, through the time of arrival (TOA) and the real distance relationship, establish the model of measurement distance and physical distance. The model can process effectively the problem of complicated structure in ultra wideband positioning system. Simulation results show that, compared with other algorithms, the proposed scheme not only inherits the advantages of LS algorithm and PSO algorithm, but also achieve high positioning accuracy in a complex environment. This paper is of great practical value and economic value of LBS.

\section{Acknowledgement}

In this paper, the research was sponsored by the Academician workstation cooperation in science and technology project of Henan Province (Project No.122106000049) and Talent Team Construction Plan of Science and Technology (Project No. 131PCXTD594).

\section{References}

[1]EIJI OKAMOTO;MANATO HORIBA.Particle swarm optimization based low complexity three dimensional UWB localization scheme.ICUFN 2014-6th International Conference on Ubiquitous and Future Networks,2014[C].Washington:IEEE Computer soci- ety:120-124.

[2]MAURICE CLERC.Particle Swarm Optimozation[M].New Jersey:Wiley,2013:13-239.

[3]XIN LI;FUCHENG CAO.Location Based TOA Algorithm for UWB Wireless Body Area Networks: 2014 IEEE 12th International Conference on Dependable, Autonomic and Secure Computing.2014[C]. Washington: IEEE Computer Society, 2014: 507-511.

[4]REZAIESARLAK,REZA;MANTEGHI,MAJID:A space-frequency technique for chipless RFID tag localization[J].IEEE Transactions on Antennas and Propagation,2014,62(11):5790-5797.

[5]WANG QING;CHEN FENG.Object tracking via partia-l Least squares analysis[J].IEEE Transactions on Image Processing,2012,21(10):4454-4465.

[6]J.XU;M.MA;C.L.LAW.Performance of time-difference-of-arrival ultra wideband indoor localisation [J].IET Science, Measurement and Technology 2011, 5(2):46-53.

[7]ZWIRELLO LUKASZ;SCHIPPER TOM;JALILVAND MALYHWE. Realization limits of impulse-based localization system for large-scale indoor applications [J]. IEEE Transactions on Instrumentation and Measurement 2015,64(1):39-51.

[8] BIN XU;GUODONG SUN;RAN YU.High Accuracy TDOA Based Localization without Time Synchronization[J]. IEEE Transactions on Parallel and Distributed Systems 2013, 24 (8) :1567-1576.

[9]K.C.HO.Bias Reduction for an Explicit Solution of Source Localization Using TDOA[J].IEEE Transactions on Signal Processing 2012,60(5):2101-2114.

[10]HE JIE;YU YANWEI;LIU FEI.A query-driven TOA-based indoor geolocation system using smart phone[J]. Journal of Convergence Information Technology 2012,7(18):1-10.

[11]SHEN JUNYANG;MOLISCH ANDREAS F;SALMI JUSSI.Accurate passive location using TOA measurements [J]. IEEE Transactions on Wireless Communications, 2012,11(6):2182-2192. 